\title{
Nonlinear Optical Properties of Spheroidal Metallic Inclusions in a Dielectric Medium
}

\author{
Bernard de Dormale ${ }^{1}$ and Vo-Van Truong ${ }^{2}$ \\ ${ }^{1}$ Département de Mathématiques et de Statistique, Université de Moncton, Moncton, NB, Canada E1A 3E9 \\ ${ }^{2}$ Department of Physics, Concordia University, 7141 Sherbrooke West, SP 367.03, Montréal, QC, Canada H4B 1R6
}

Correspondence should be addressed to Vo-Van Truong, tvovan@alcor.concordia.ca

Received 23 August 2011; Accepted 27 September 2011

Academic Editors: B. Coasne and A. Ryasnyanskiy

Copyright (C) 2011 B. de Dormale and V.-V. Truong. This is an open access article distributed under the Creative Commons Attribution License, which permits unrestricted use, distribution, and reproduction in any medium, provided the original work is properly cited.

\begin{abstract}
A model for linear and nonlinear optical properties of a composite material consisting of spheroidal metal inclusions embedded in a host medium has been formulated using an effective medium approach. Both aligned and randomly oriented spheroids have been considered, and the results obtained showed a considerable difference between the two situations. Numerical calculations for metallic Au inclusions in a glass matrix have shown that the linear absorption in the case of aligned spheroids with their symmetry axis parallel to the $z$-axis is largely dependent on the depolarization factor, exhibiting an absorption in the vicinity of $500 \mathrm{~nm}$ when the depolarization factor in the direction parallel to the rotational symmetry axis is small. This structure shifts progressively to higher wavelengths when this depolarization factor is increased. In the case of randomly oriented spheroids, contributions from the different particle depolarization factors are present and prominent structures in the linear absorption appear in the long wavelength region, beyond $700 \mathrm{~nm}$. Nonlinear optical properties for both aligned and randomly oriented spheroids also show a strong dependence on the depolarization factor and significant enhancements of these properties can be observed, suggesting possible tailoring of composite properties for various applications.
\end{abstract}

\section{Introduction}

Nonlinear optical properties of composite optical materials consisting of metallic inclusions in a dielectric medium that exhibit large nonlinearities and fast responses are very attractive due to their great potential for various applications in the photonic and nanotechnology field [1-3]. As the metallic particles in many cases could be approximated by very small spheres, most of the theoretical treatments have been based on this shape assumption [4-6]. Sipe and Boyd [6], for example, calculated the nonlinear susceptibility of a composite comprised of spherical inclusion particles within a host material using the Maxwell Garnett model, while Agarwal and Gupta [4] adopted a T-matrix approach to the problem.

As progress in nanofabrication has made possible the production of nanoparticles of various shapes, it is highly desirable to consider the possibilities offered by the shape dependence of the nonlinear optical properties. This will effectively allow the tailoring of optical properties according to specific needs and widening the range of applications. A previous study by $\mathrm{Wu}$ et al. [7] examined the case of spheroidal metal particles embedded in a host medium using decoupling approximation and spectral representation. It was found that the effective nonlinear susceptibility for such composites indeed showed a strong dependence on the particle shape via the depolarization factor. For simplicity it was assumed that all spheroidal metal particles were oriented parallel to one another with the rotationally symmetric axes lying in the $z$-axis.

In the present work, we use rather an effective medium approach in a similar manner as in [4] and study the influence of the depolarization factor of spheroidal metal particles on the linear absorption as well as on the nonlinear optical properties. Moreover, two particle configurations are considered here. We first assume that all particles are aligned, that is, all the spheroids have the same orientation, for example, that their axis of symmetry are all parallel to the $z$-axis. In a second instance, we assume that the orientation of the spheroids 
is random: this means that the polarizations must be replaced by average polarizations, the averaging being over all possible orientations of the particles. As an illustration, numerical simulations are conducted for $\mathrm{Au}$ spheroidal inclusions in a glass matrix for a fill factor varying from 0.05 to 0.20 and for various values of the depolarization factor, covering the shape range of oblate and prolate spheroids. As expected, a strong dependence of the linear and nonlinear optical properties on the particle shape can be established, but we also find that there is a considerable difference between aligned and randomly oriented spheroids.

\section{Effective Medium Theory}

We consider the case of small inclusions in an infinite medium of dielectric constant $\varepsilon_{h}$ and the context of quasistatic approximation when the size of the inclusions is much smaller than the wavelength of the incident light. There are various ways to establish that, if the quasistatic approximation is valid and if we assume that the inclusions are homogenously distributed, the field acting on each inclusion is given by

$$
\mathbf{E}_{0}=\mathbf{E}+\frac{4 \pi}{3 \varepsilon_{h}} \mathbf{P}
$$

where $\mathbf{E}$ is the macroscopic field and $\mathbf{P}$ is the macroscopic polarization. Since we must have

$$
\mathbf{P}=\frac{\varepsilon_{e}-\varepsilon_{h}}{4 \pi} \mathbf{E},
$$

where $\varepsilon_{e}$ is the effective dielectric constant of the composite medium, it results that, in the case of linear polarization,

$$
\mathbf{E}_{0}=q \mathbf{E}, \quad q=\frac{\varepsilon_{e}+2 \varepsilon_{h}}{3 \varepsilon_{h}}
$$

If we now assume that the inclusions have a linear polarizability $\eta^{(1)}$ and nonlinear polarizabilities $\eta_{a}^{(3)}$ and $\eta_{b}^{(3)}$, we must have in first approximation that

$$
\begin{aligned}
\mathbf{P}_{L}+\mathbf{P}_{N L}= & \frac{\eta^{(1)}}{V}\left(\mathbf{E}+\frac{4 \pi}{3 \varepsilon_{h}} \mathbf{P}_{L}+\frac{4 \pi}{3 \varepsilon_{h}} \mathbf{P}_{N L}\right) \\
& +\frac{q|q|^{2}}{V}\left(\eta_{a}^{(3)}\left(\mathbf{E} \cdot \mathbf{E}^{*}\right) \mathbf{E}+\eta_{b}^{(3)}(\mathbf{E} \cdot \mathbf{E}) \mathbf{E}^{*}\right),
\end{aligned}
$$

where $V$ is the average volume of the host material per inclusion. Thus,

$$
\begin{gathered}
\left(1-\frac{4 \pi \eta^{(1)}}{3 \varepsilon_{h} V}\right) \mathbf{P}_{L}=\frac{\eta^{(1)}}{V} \mathbf{E}, \\
\left(1-\frac{4 \pi \eta^{(1)}}{3 \varepsilon_{h} V}\right) \mathbf{P}_{N L}=\frac{q|q|^{2}}{V}\left(\eta_{a}^{(3)}\left(\mathbf{E} \cdot \mathbf{E}^{*}\right) \mathbf{E}+\eta_{b}^{(3)}(\mathbf{E} \cdot \mathbf{E}) \mathbf{E}^{*}\right) .
\end{gathered}
$$

Since

$$
\mathbf{P}_{L}=\frac{q \eta^{(1)}}{V} \mathbf{E}
$$

it results that

$$
1-\frac{4 \pi \eta^{(1)}}{3 \varepsilon_{h} V}=\frac{1}{q}
$$

This leads to the Maxwell Garnett-type relation [8]

$$
1-\frac{1}{q}=\frac{\varepsilon_{e}-\varepsilon_{h}}{\varepsilon_{e}+2 \varepsilon_{h}}=\frac{4 \pi \eta^{(1)}}{3 \varepsilon_{h} V}
$$

and to

$$
\mathbf{P}_{N L}=\frac{q^{2}|q|^{2}}{V}\left(\eta_{a}^{(3)}\left(\mathbf{E} \cdot \mathbf{E}^{*}\right) \mathbf{E}+\eta_{b}^{(3)}(\mathbf{E} \cdot \mathbf{E}) \mathbf{E}^{*}\right),
$$

which means that the nonlinear coefficients of the effective medium will be

$$
A=q^{2}|q|^{2} \frac{\eta_{a}^{(3)}}{V}, \quad B=2 q^{2}|q|^{2} \frac{\eta_{b}^{(3)}}{V} .
$$

\section{Aligned Spheroids}

Let us now consider inclusions that are spheroids with semimajor axes $a=b$ and $c$. We will first assume that all the spheroids have the same orientation, for example, that their axes of symmetry are all parallel to the $z$ axis. In that case

$$
\mathbf{E}_{i}=\left(\mathbf{I}+\frac{\varepsilon_{i}-\varepsilon_{h}}{\varepsilon_{h}} \mathbf{L}\right)^{-1} \mathbf{E},
$$

where $\mathbf{I}$ is the identity tensor and $\mathbf{L}$ is the diagonal tensor

$$
\mathbf{L}=\left(\begin{array}{ccc}
L_{x} & 0 & 0 \\
0 & L_{y} & 0 \\
0 & 0 & L_{z}
\end{array}\right),
$$

with

$L_{z}=\frac{1-e^{2}}{2 e^{3}}\left(\ln \frac{1+e}{1-e}-2 e\right) \approx \frac{1}{3}-\frac{2}{15} e^{2}, \quad e=\sqrt{1-\left(\frac{a}{c}\right)^{2}}$

for oblate spheroids and

$$
L_{z}=\frac{1+e^{2}}{e^{3}}\left(e-\tan ^{-1} e\right) \approx \frac{1}{3}+\frac{2}{15} e^{2}, \quad e=\sqrt{\left(\frac{a}{c}\right)^{2}-1}
$$

for prolate spheroids, while

$$
L_{x}=L_{y}=\frac{1-L_{z}}{2} .
$$

Equation (11) gives us very valuable information: the uniform polarization is

$$
\mathbf{P}_{i}=\frac{\varepsilon_{i}-\varepsilon_{h}}{4 \pi} \mathbf{E}_{i}
$$

and the electric field generated by this uniform polarization is

$$
\mathbf{E}_{i}-\mathbf{E}=-\frac{\varepsilon_{i}-\varepsilon_{h}}{\varepsilon_{h}} \mathbf{L E}_{i}=-\frac{4 \pi}{\varepsilon_{h}} \mathbf{L} \mathbf{P}_{i}
$$


This means that a constant polarization $\mathbf{P}$ inside the spheroid generates a constant electric field equal to $-\left(4 \pi / \varepsilon_{h}\right) \mathbf{L P}$.

Let us now suppose that the medium of the spheroid has a nonlinear response characterized by parameters $A_{i}$ and $B_{i}$, that is, the field $\mathbf{E}_{i}$ would induce in the bulk medium a polarization

$$
\mathbf{P}_{i, N L}^{(\infty)}=A_{i}\left(\mathbf{E}_{i} \cdot \mathbf{E}_{i}^{*}\right) \mathbf{E}_{i}+\frac{1}{2} B_{i}\left(\mathbf{E}_{i} \cdot \mathbf{E}_{i}\right) \mathbf{E}_{i}^{*} .
$$

We will then observe a total nonlinear polarization $\mathbf{P}_{i, N L}$ and electric field $\mathbf{E}_{i, N L}$ that must be related by

$$
\mathbf{P}_{i, N L}=\mathbf{P}_{i, N L}^{(\infty)}+\frac{\varepsilon_{i}-\varepsilon_{h}}{4 \pi} \mathbf{E}_{i, N L} .
$$

Since we must also have

$$
\mathbf{E}_{i, N L}=-\frac{4 \pi}{\varepsilon_{h}} \mathbf{L} \mathbf{P}_{i, N L},
$$

we can conclude that

$$
\mathbf{P}_{i, N L}=\mathbf{P}_{i, N L}^{(\infty)}-\frac{\varepsilon_{i}-\varepsilon_{h}}{\varepsilon_{h}} \mathbf{L} \mathbf{P}_{i, N L},
$$

that is,

$$
\mathbf{P}_{i, N L}=\left(\mathbf{I}+\frac{\varepsilon_{i}-\varepsilon_{h}}{\varepsilon_{h}} \mathbf{L}\right)^{-1} \mathbf{P}_{i, N L}^{(\infty)} .
$$

For sake of simplicity, we will limit our considerations in this section to the special cases where $\mathbf{E}$ and $\mathbf{E}^{*}$ are either both pointing in the $z$ direction or both parallel to the $x y$ plane. If we then define

$$
\alpha=\frac{\varepsilon_{h}}{\varepsilon_{h}+\left(\varepsilon_{i}-\varepsilon_{h}\right) L_{i}},
$$

where $L_{i}=L_{z}$ or $L_{i}=L_{x}$, depending on the case, we will have

$$
\mathrm{E}_{i}=\alpha \mathbf{E}
$$

so that

$$
\begin{gathered}
\mathbf{P}_{i}=\frac{\varepsilon_{i}-\varepsilon_{h}}{4 \pi} \alpha \mathbf{E}, \\
\mathbf{P}_{i, N L}=\alpha^{2}|\alpha|^{2}\left(A_{i}\left(\mathbf{E} \cdot \mathbf{E}^{*}\right) \mathbf{E}+\frac{1}{2} B_{i}(\mathbf{E} \cdot \mathbf{E}) \mathbf{E}^{*}\right) .
\end{gathered}
$$

This leads to the linear and nonlinear dipole moments

$$
\begin{gathered}
\mathbf{p}=\frac{a^{2} c}{3}\left(\varepsilon_{i}-\varepsilon_{h}\right) \alpha \mathbf{E} \\
\mathbf{p}_{N L}=\frac{4 \pi a^{2} c}{3} \alpha^{2}|\alpha|^{2}\left(A_{i}\left(\mathbf{E} \cdot \mathbf{E}^{*}\right) \mathbf{E}+\frac{1}{2} B_{i}(\mathbf{E} \cdot \mathbf{E}) \mathbf{E}^{*}\right) .
\end{gathered}
$$

The linear and nonlinear polarizabilities are thus

$$
\begin{gathered}
\eta^{(1)}=\frac{a^{2} c}{3}\left(\varepsilon_{i}-\varepsilon_{h}\right) \alpha, \\
\eta_{a}^{(3)}=\frac{4 \pi a^{2} c}{3} \alpha^{2}|\alpha|^{2} A_{i}, \\
\eta_{b}^{(3)}=\frac{2 \pi a^{2} c}{3} \alpha^{2}|\alpha|^{2} B_{i} .
\end{gathered}
$$

These values, combined with the results of Section 2, give the Maxwell Garnett-like result

$$
\frac{\varepsilon_{e}}{\varepsilon_{h}}=\frac{L_{i} \varepsilon_{i}+\left(1-L_{i}\right) \varepsilon_{h}+(2 / 3) f\left(\varepsilon_{i}-\varepsilon_{h}\right)}{L_{i} \varepsilon_{i}+\left(1-L_{i}\right) \varepsilon_{h}-(1 / 3) f\left(\varepsilon_{i}-\varepsilon_{h}\right)}
$$

as well as the values

$$
A=f(\alpha q)^{2}|\alpha q|^{2} A_{i}, \quad B=f(\alpha q)^{2}|\alpha q|^{2} B_{i},
$$

with

$$
\alpha q=\frac{\varepsilon_{h}}{L_{i} \varepsilon_{i}+\left(1-L_{i}\right) \varepsilon_{h}-(1 / 3) f\left(\varepsilon_{i}-\varepsilon_{h}\right)} .
$$

For spheres, these results are identical with those of Sipe and Boyd [6].

\section{Enhancement of Nonlinear Properties}

One would like to know how, for a given fill factor, the specific shape of inclusions influences the linear and nonlinear properties of the composite medium. For oblate spheroids, one has $0 \leq e<1$ and $L_{z}$ varies from $1 / 3$ for a sphere $(e=0)$ to 0 when $e \rightarrow 1$ and the spheroid becomes needle-like. The corresponding range for $L_{x}$ is from $1 / 3$ to $1 / 2$. For prolate spheroids, one has $e \geq 0$ and $L_{z}$ varies from $1 / 3$ for a sphere $(e=0)$ to 1 when $e \rightarrow \infty$ and the spheroid is almost a disk, with corresponding range for $L_{x}$ being from $1 / 3$ to 0 .

In order to assert how the different values of the depolarizing factor can influence the linear properties of the composite medium, we have computed the linear absorption factor $\alpha$ and the ratio $A /\left|A_{i}\right|=B /\left|B_{i}\right|$ for gold inclusions in glass for fill-factor values $f$ of $0.05,0.1$, and 0.2 . These latter values have been chosen because they reflected well-experimental values of gold composites and effective medium approaches were known to be widely accepted in the limit of small fill factors. Linear optical constants for $\mathrm{Au}$ were obtained from [9]. The results of the computations are given in Figures 1 and 2.

The results show that nonlinear optical properties can be significantly increased for oblate spheroids when the field is parallel to the rotation axis. For prolate spheroids, the increase is obtained when the field is perpendicular to the rotation axis. In both cases, however, there is also an equally significant increase in the linear absorption factor.

\section{Randomly Oriented Spheroids}

We will now consider the case where the orientation of the spheroids is random: this means that the polarizations must be replaced by average polarizations, with the averaging being over all possible orientations. To achieve that, we will consider two system of coordinates, one (unprimed) attached to the host medium and one (primed) attached to the spheroid, and will again assume that the axis of symmetry of the spheroid coincides with the axis $O z^{\prime}$. We will use a prime 


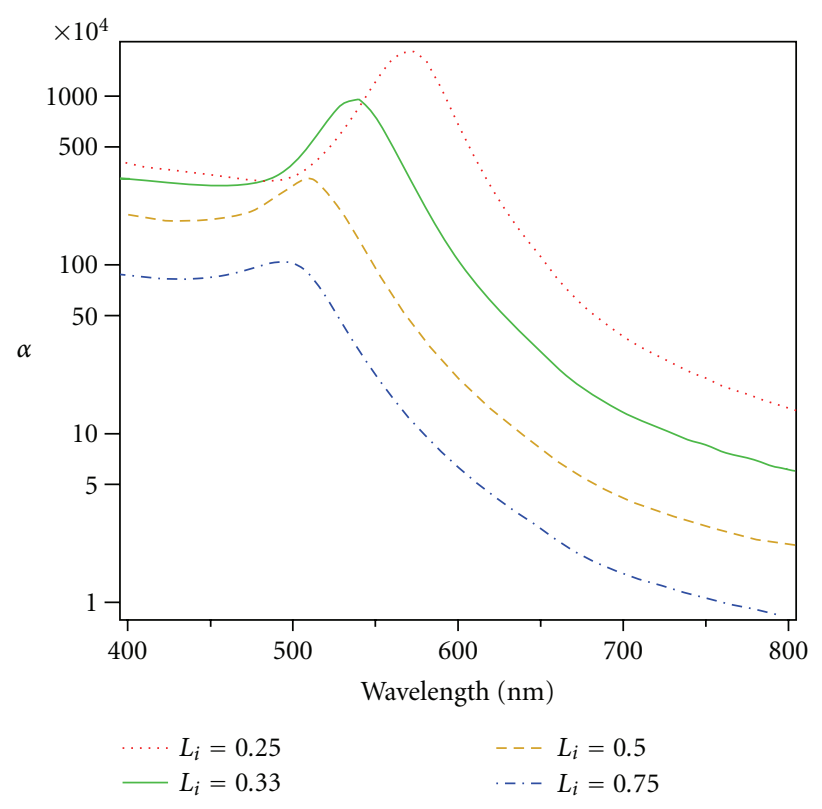

(a)

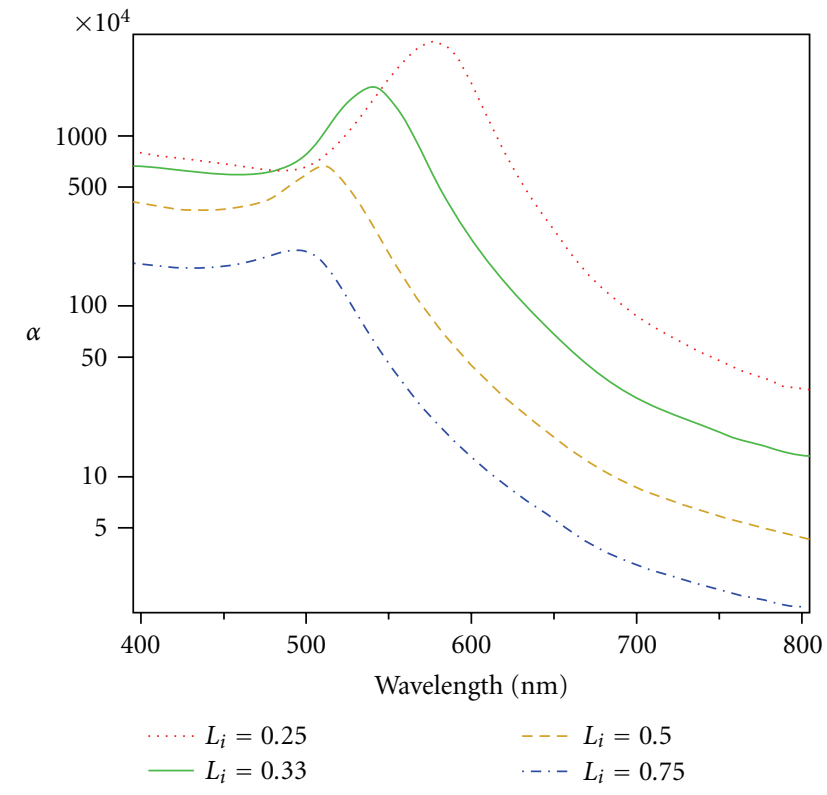

(b)

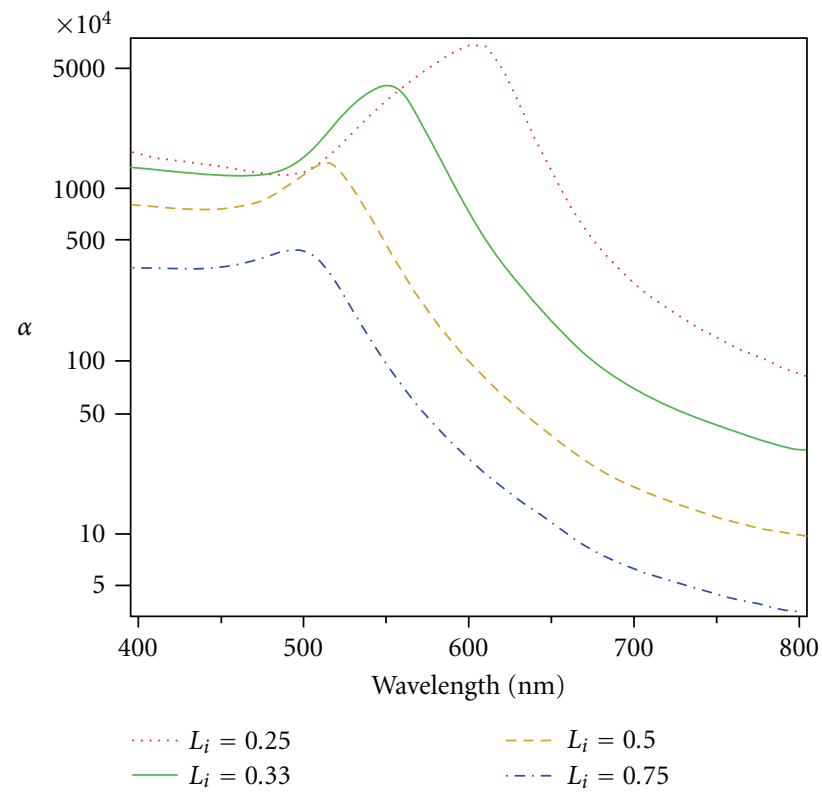

(c)

Figure 1: Linear absorption factor $\alpha$ as a function of the depolarization factor $L_{i}$ and the fill factor $f((\mathrm{a}): f=0.05,(\mathrm{~b}): f=0.10$, and $(\mathrm{c})$ : $f=0.2$ ): aligned spheroids of $\mathrm{Au}$ in glass.

to indicate the components of a vector or tensor in the primed system, so that (1) now becomes

$$
\mathbf{E}_{i}^{\prime}=\left(\mathbf{I}+\frac{\varepsilon_{i}-\varepsilon_{h}}{\varepsilon_{h}} \mathbf{L}^{\prime}\right)^{-1} \mathbf{E}^{\prime},
$$

where the notation $\mathbf{L}^{\prime}$ is used for the diagonal tensor of the preceding section.

(Of course, the unit tensor has the same components in both systems, so that we do not add a prime.)
Note that if we start with the axis $O z^{\prime}$ parallel to the axis $\mathrm{O} z$, because of the axial symmetry of the spheroid, we only need two Euler angles to give it an arbitrary orientation. Taking those angles as the polar angles of the axis $O z^{\prime}$ in the unprimed system, we will have

$$
\begin{gathered}
\mathbf{E}_{i}=\mathbf{R}(\theta, \phi) \mathbf{E}_{i}^{\prime}, \\
\mathbf{L}=\mathbf{R}(\theta, \phi) \mathbf{L}^{\prime} \mathbf{R}^{T}(\theta, \phi),
\end{gathered}
$$




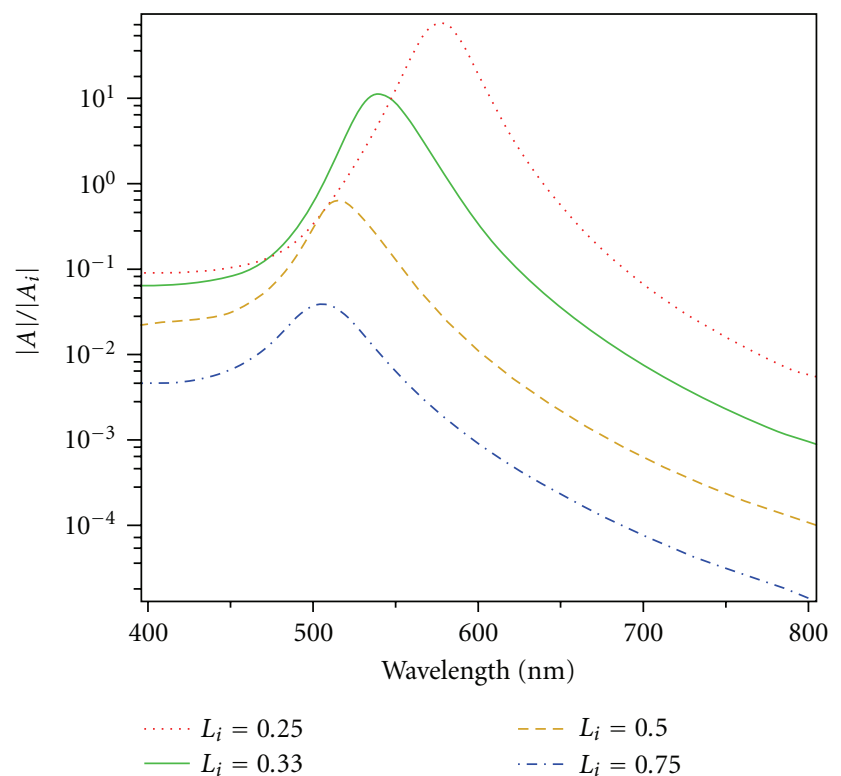

(a)

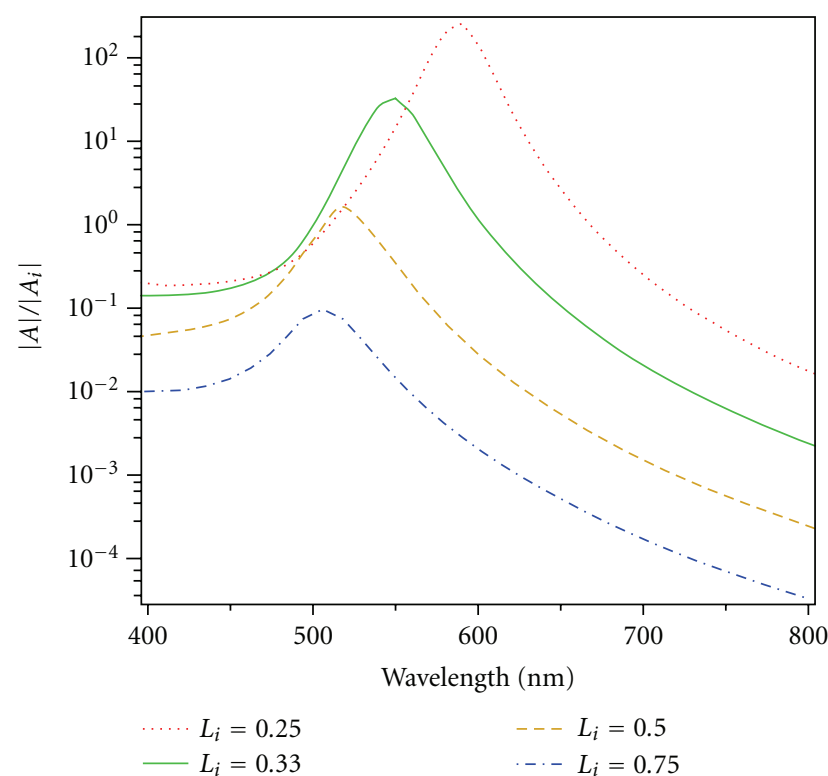

(b)

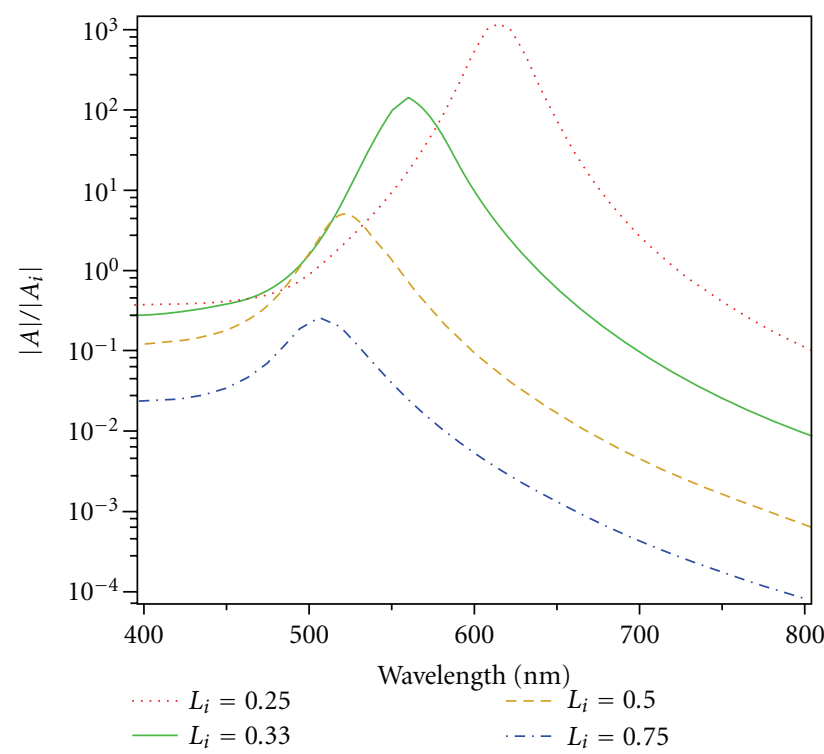

(c)

Figure 2: Ratio $A / A_{i}$ as a function of the depolarization factor $L_{i}$ and the fill factor $f((\mathrm{a}): f=0.05,(\mathrm{~b}): f=0.10$, and $(\mathrm{c}): f=0.2):$ aligned spheroids of $\mathrm{Au}$ in glass.

where

$$
\mathbf{R}(\theta, \phi)=\left(\begin{array}{ccc}
\cos \phi & -\sin \phi & 0 \\
\sin \phi & \cos \phi & 0 \\
0 & 0 & 1
\end{array}\right)\left(\begin{array}{ccc}
\cos \theta & 0 & \sin \theta \\
0 & 1 & 0 \\
-\sin \theta & 0 & \cos \theta
\end{array}\right)
$$

\section{Average Polarizabilities}

In order to compute the linear and nonlinear average polarizabilities of the spheroid, we must now compute the linear and nonlinear dipole moments of the spheroid in the unprimed system of coordinates and average them over all possible orientations of the particle.

If $Q$ is a physical quantity depending on the angles $\theta$ and $\varnothing$, we will define its average value $\langle Q\rangle$ by

$$
\langle Q\rangle=\int_{0}^{2 \pi} \int_{0}^{\pi} Q \sin \theta d \theta d \phi .
$$

Note that if $\mathbf{T}$ is a tensor that would be diagonal in the primed coordinate system, that is,

$$
\mathbf{T}^{\prime}=\left(\begin{array}{ccc}
T_{x} & 0 & 0 \\
0 & T_{y} & 0 \\
0 & 0 & T_{z}
\end{array}\right),
$$


with $T_{y}=T_{x}$, then we can write

$$
\mathbf{T}^{\prime}=T_{x} \mathbf{I}+\left(T_{z}-T_{x}\right) \mathbf{N}^{\prime}
$$

where

$$
\mathbf{N}^{\prime}=\left(\begin{array}{lll}
0 & 0 & 0 \\
0 & 0 & 0 \\
0 & 0 & 1
\end{array}\right)
$$

Thus,

$$
\mathbf{T}=T_{x} \mathbf{I}+\left(T_{z}-T_{x}\right) \mathbf{N}
$$

where

$$
\mathbf{N}=\mathbf{R}(\theta, \phi) \mathbf{N}^{\prime} \mathbf{R}^{T}(\theta, \phi)
$$

has the property that for any vector $\mathbf{u}$

$$
\mathbf{N u}=(\mathbf{n} \cdot \mathbf{u}) \mathbf{n}, \quad \mathbf{n}=(\sin \theta \cos \phi, \sin \theta \sin \phi, \cos \theta) .
$$

Noting that

$$
\begin{gathered}
\mathbf{n} \cdot \mathbf{u}=\frac{1}{2}\left[\left(u_{1}-i u_{2}\right) e^{i \varphi}+\left(u_{1}+i u_{2}\right) e^{-i \varphi}\right] \sin \theta+u_{3} \cos \theta \\
\left(u_{1}-i u_{2}\right)\left(v_{1}+i v_{2}\right)+\left(u_{1}+i u_{2}\right)\left(v_{1}+i v_{2}\right)=2\left(u_{1} v_{1}+u_{2} v_{2}\right),
\end{gathered}
$$

it is easily seen that

$$
\begin{gathered}
\langle\mathbf{N u}\rangle=\frac{1}{3} \mathbf{u} \\
\langle(\mathbf{u} \cdot \mathbf{N v}) \mathbf{N} \mathbf{w}\rangle=\frac{1}{15}[(\mathbf{u} \cdot \mathbf{v}) \mathbf{w}+(\mathbf{u} \cdot \mathbf{w}) \mathbf{v}+(\mathbf{v} \cdot \mathbf{w}) \mathbf{u}] .
\end{gathered}
$$

The linear and nonlinear polarizabilities are

$$
\mathbf{P}_{i}=\frac{\varepsilon_{i}-\varepsilon_{h}}{4 \pi}(\alpha \mathbf{I}+\beta \mathbf{N}) \mathbf{E}
$$

where

$$
\begin{aligned}
\alpha= & \frac{\varepsilon_{h}}{\varepsilon_{h}+\left(\varepsilon_{i}-\varepsilon_{h}\right) L_{x}}, \\
\beta= & \frac{\varepsilon_{h}}{\varepsilon_{h}+\left(\varepsilon_{i}-\varepsilon_{h}\right) L_{z}}-\frac{\varepsilon_{h}}{\varepsilon_{h}+\left(\varepsilon_{i}-\varepsilon_{h}\right) L_{x}}, \\
\mathbf{P}_{i, N L}= & A_{i}\left[\mathbf{E} \cdot(|\gamma| \mathbf{I}+|\delta| \mathbf{N}) \mathbf{E}^{*}\right](\gamma \mathbf{I}+\delta \mathbf{N}) \mathbf{E} \\
& +\frac{1}{2} B_{i}[\mathbf{E} \cdot(\gamma \mathbf{I}+\delta \mathbf{N}) \mathbf{E}](|\gamma| \mathbf{I}+|\delta| \mathbf{N}) \mathbf{E}^{*},
\end{aligned}
$$

where

$$
\begin{gathered}
\gamma=\left(\frac{\varepsilon_{h}}{\varepsilon_{h}+\left(\varepsilon_{i}-\varepsilon_{h}\right) L_{x}}\right)^{2}, \\
\delta=\left(\frac{\varepsilon_{h}}{\varepsilon_{h}+\left(\varepsilon_{i}-\varepsilon_{h}\right) L_{z}}\right)^{2}-\left(\frac{\varepsilon_{h}}{\varepsilon_{h}+\left(\varepsilon_{i}-\varepsilon_{h}\right) L_{x}}\right)^{2} .
\end{gathered}
$$

This leads to the linear and nonlinear dipole moments

$$
\begin{aligned}
& \mathbf{p}=\frac{a^{2} c}{3}\left(\varepsilon_{i}-\varepsilon_{h}\right)(\alpha \mathbf{I}+\beta \mathbf{N}) \mathbf{E}, \\
& \mathbf{p}_{N L}=\frac{4 \pi a^{2} c}{3}\left\{A_{i}\left[\mathbf{E} \cdot(|\gamma| \mathbf{I}+|\delta| \mathbf{N}) \mathbf{E}^{*}\right](\gamma \mathbf{I}+\delta \mathbf{N}) \mathbf{E}\right. \\
& \left.+\frac{1}{2} B_{i}[\mathbf{E} \cdot(\gamma \mathbf{I}+\delta \mathbf{N}) \mathbf{E}](|\gamma| \mathbf{I}+|\delta| \mathbf{N}) \mathbf{E}^{*}\right\} ;
\end{aligned}
$$

so that after averaging, we obtain

$$
\begin{aligned}
& \eta^{(1)}=\frac{a^{2} c}{3}\left(\varepsilon_{i}-\varepsilon_{h}\right)\left(\alpha+\frac{\beta}{3}\right), \\
& \eta_{a}^{(3)}=\frac{4 \pi a^{2} c}{3}\left[\left(\gamma|\gamma|+\frac{\gamma|\delta|+|\gamma| \delta}{3}+\frac{2 \delta|\delta|}{15}\right) A_{i}+\frac{\delta|\delta|}{15} B_{i}\right], \\
& \eta_{b}^{(3)}=\frac{2 \pi a^{2} c}{3}\left[\left(\gamma|\gamma|+\frac{\gamma|\delta|+|\gamma| \delta}{3}+\frac{\delta|\delta|}{15}\right) B_{i}+\frac{2 \delta|\delta|}{15} A_{i}\right] .
\end{aligned}
$$

Note again that for sphere, $L_{x}=L_{y}=L_{z}=1 / 3$, so that

$$
\alpha=\frac{3 \varepsilon_{h}}{\varepsilon_{i}+2 \varepsilon_{h}}, \quad \gamma=\alpha^{2}, \quad \beta=\delta=0 .
$$

Thus, in this case, our results remain identical with those of Sipe and Boyd [6].

\section{Results for Randomly Oriented Spheroids}

We now present results obtained similarly to those in Section 4 for aligned spheroids. Since $A$ is now a function of $B_{i}$ and $B$ of $A_{i}$, we have, for simplicity, assumed that $B_{i}=A_{i}$. The results for randomly oriented spheroids are given in Figures 3 and 4.

Examination of the linear absorption factor for randomly oriented spheroids given in Figure 3 will reveal a drastic difference, except for the case of the spherical particles $\left(L_{z}=\right.$ 0.33 ), with the aligned spheroids (Figure 1). When $L_{z}=0.25$ (oblate spheroids), the random orientation of the spheroids leads to a broadening of the absorption peak with the addition of a shoulder near $530 \mathrm{~nm}$. For $L_{z}$ at 0.5 and 0.75 (prolate spheroids), the appearance of a prominent peak at longer wavelengths can be noted. This particular peak would be attributed to the contributions from the depolarization factors $L_{x}$ and $L_{y}\left(L_{x}=L_{y}\right)$ that are, respectively, 0.25 and 0.125 for the two values of $L_{z}$ mentioned.

The nonlinear optical properties exhibit also significant enhancements (Figure 4). It is noted that when $L_{z}$ is equal to 0.25 , the shoulder at $530 \mathrm{~nm}$ appears more clearly than in Figure 3.

\section{Conclusion}

A model for linear and nonlinear optical properties of a composite material consisting of spheroidal metal inclusions embedded in a host medium has been formulated in 


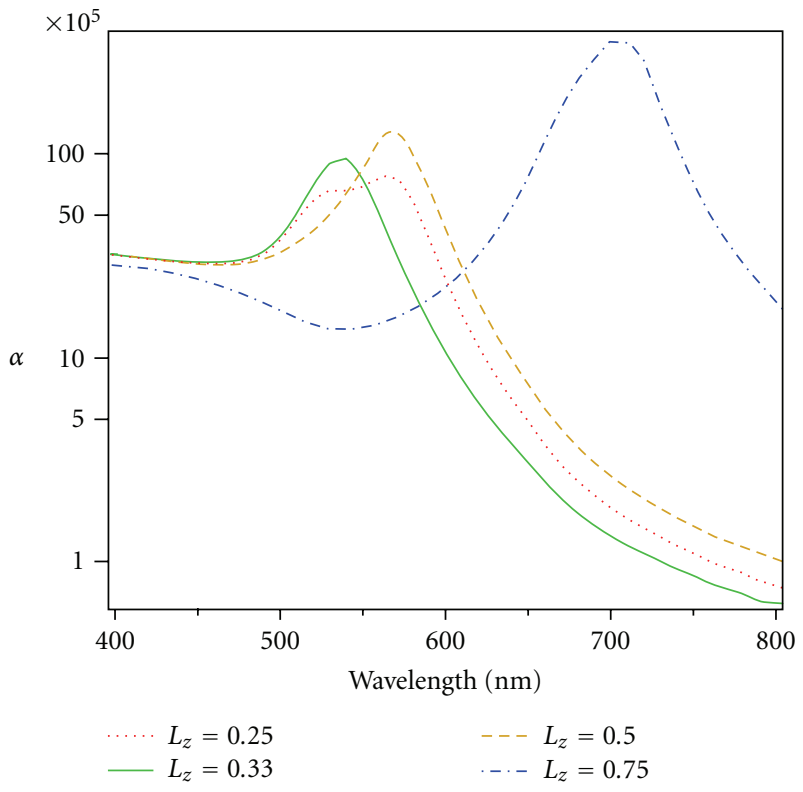

(a)

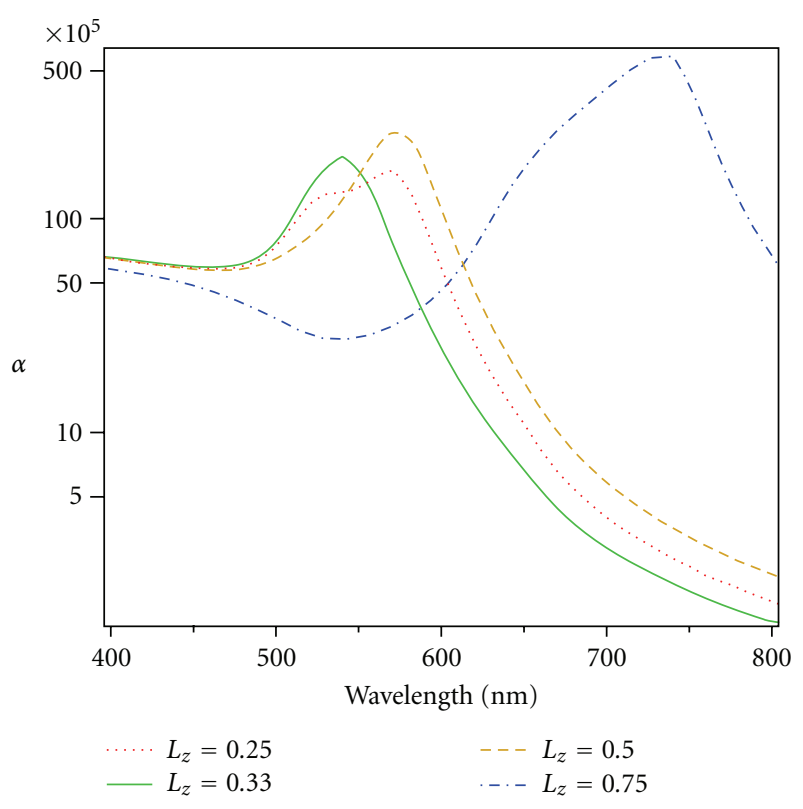

(b)

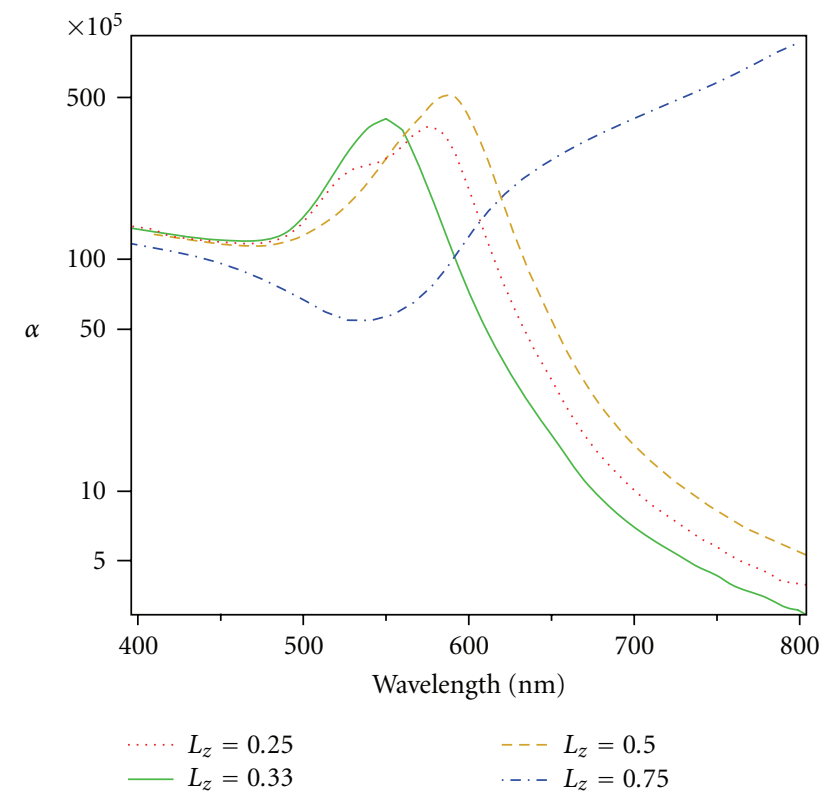

(c)

Figure 3: Linear absorption factor $\alpha$ as a function of the depolarization factor $L_{z}$ and the fill factor $f((\mathrm{a}): f=0.05$, (b): $f=0.10,(\mathrm{c})$ : $f=0.2$ ): randomly oriented spheroids of Au in glass.

the present work using an effective medium approach. Both aligned and randomly oriented spheroids have been treated, showing a considerable difference between the two situations. Numerical simulations performed for Au inclusions in glass have shown that the linear absorption in the case of aligned spheroids (with symmetry axis parallel to the $z$-axis) is largely dependent on the depolarization factor, exhibiting an absorption in the vicinity of $500 \mathrm{~nm}$ when the depolarization factor $L_{z}$ is small (e.g., 0.25). This structure shifts progressively to higher wavelengths when $L_{z}$ is increased, or alternatively when $L_{x}$ and $L_{y}$ are decreased. In the case of randomly oriented spheroids, contributions from the depolarization factors $L_{x}$ and $L_{y}$ are also present and prominent structures in the linear absorption appear in the long wavelength region. Nonlinear optical properties for both aligned and randomly oriented spheroids also show a strong dependence on the depolarization factor and significant enhancements of these properties can be observed.

The model presented here can be advantageously used for predicting the behavior of metal/dielectric composites when 


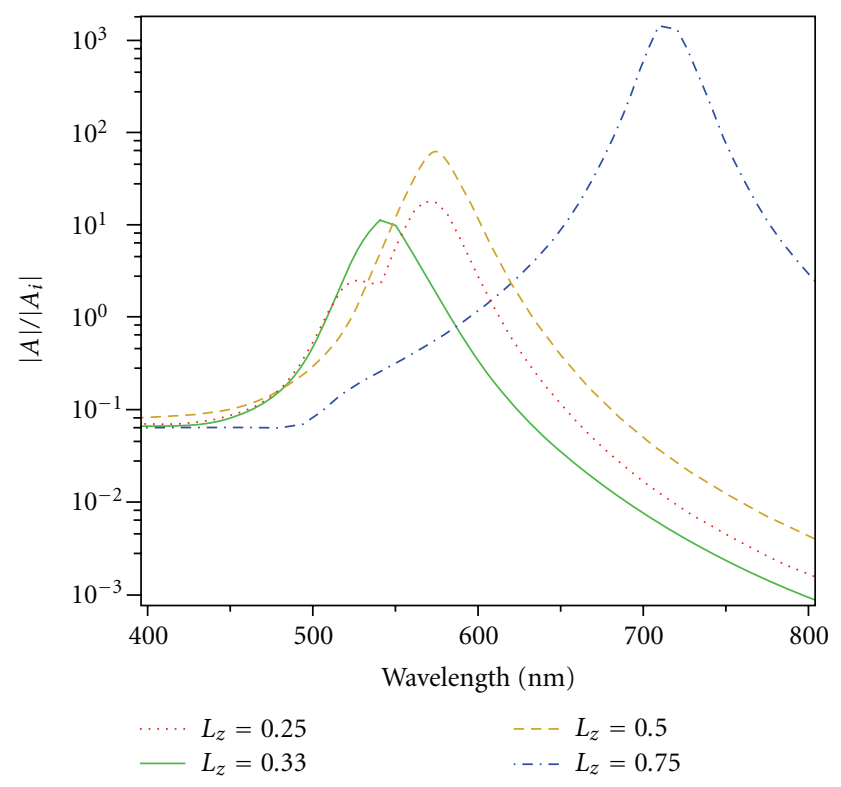

(a)

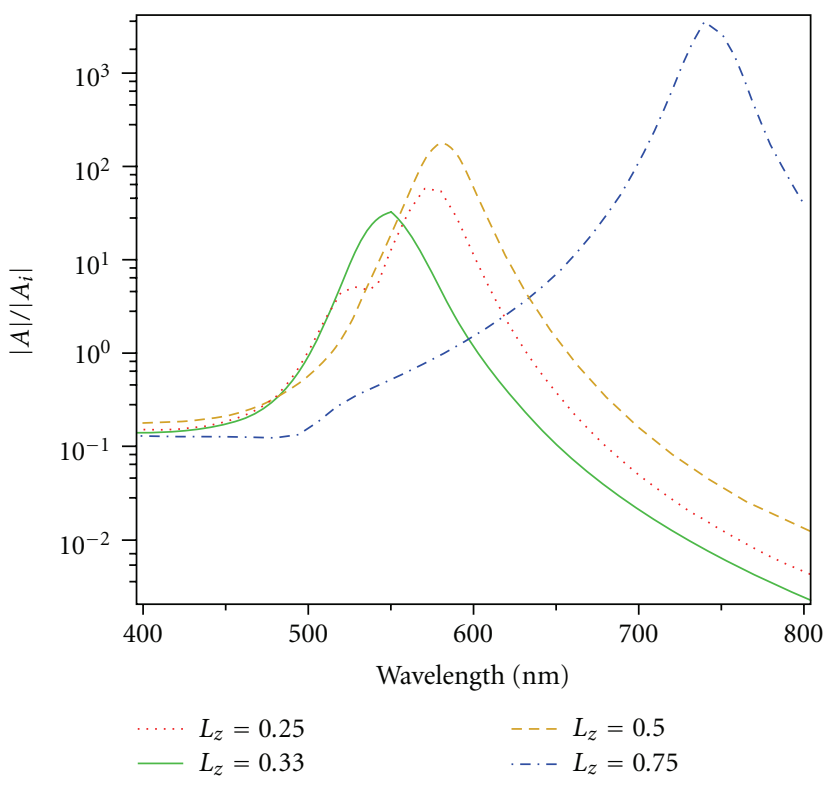

(b)

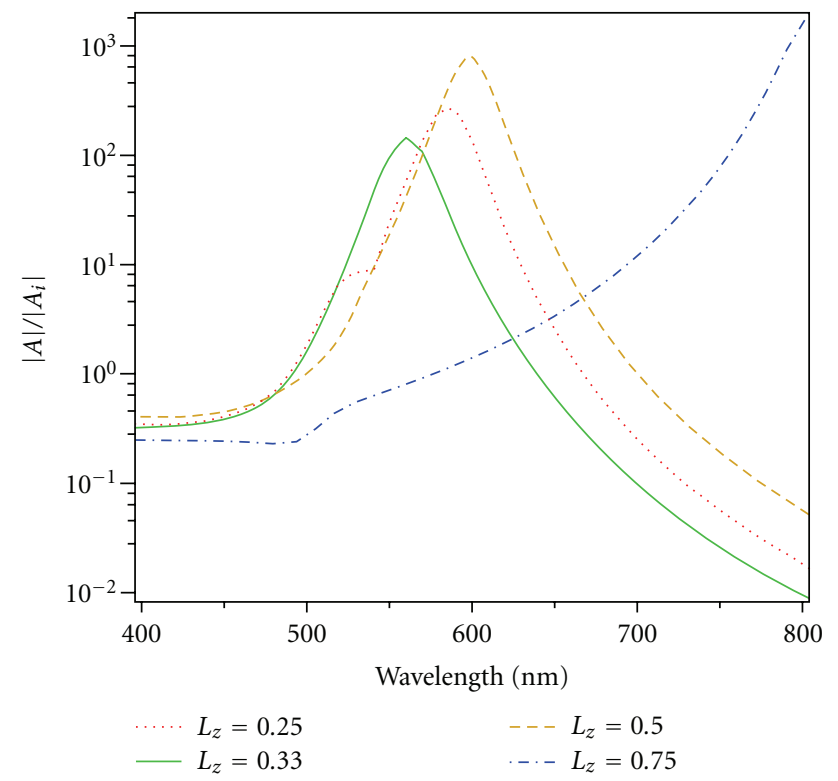

(c)

Figure 4: Ratio $A / A_{i}$ as a function of the depolarization factor $L_{z}$ and the fill factor $f((\mathrm{a}): f=0.05$, (b): $f=0.10,(\mathrm{c}): f=0.2)$ : randomly oriented spheroids of Au in glass.

the metallic inclusions are of the spheroidal shape. With progress in nanofabrication processes, tailoring of linear and nonlinear optical properties would be very much feasible.

In a subsequent paper, predictions of the present model will be compared to available experimental data.

\section{Acknowledgments}

This work has been supported the Natural Sciences and Engineering Research Council of Canada (NSERC), which is gratefully acknowledged. Thanks are also due to Professor
Georges Bader of Université de Moncton who has kindly provided the authors with the optical constants for $\mathrm{Au}$.

\section{References}

[1] H. Rigneault, J.-M. Lourtiouz, C. Delalande, and A. Leven, Nanophotonics, ISTE Ltd, Newport Beach, Calif, USA, 2006.

[2] U. Kreibig and M. Vollmer, Optical Properties of Metal Clusters, Springer, 1995.

[3] S. Qu, C. Du, Y. Song et al., "Optical nonlinearities of space selectively precipitated AU nanoparticles inside glasses," Chemical Physics Letters, vol. 356, p. 403, 2002. 
[4] G. S. Agarwal and S. D. Gupta, "T-matrix approach to the nonlinear susceptibilities of heterogeneous media," Physical Review A, vol. 38, no. 11, pp. 5678-5687, 1988.

[5] K. W. Yu and G. Q. Gu, "Effective conductivity of nonlinear composites-II. Effective-medium approximation," Physical Review B, vol. 47, no. 12, pp. 7568-7571, 1993.

[6] J. E. Sipe and R. W. Boyd, "Nonlinear susceptibility of composite optical materials in the Maxwell Garnett model," Physical Review A, vol. 46, no. 3, pp. 1614-1629, 1992.

[7] Y. M. Wu, L. Gao, and Z. Y. Li, "The influence of particle shape on nonlinear optical properties of metal-dielectric composites," Physica Status Solidi (B) Basic Research, vol. 220, no. 2, pp. 9971008, 2000.

[8] J. C. Maxwell Garnett, "Colours in metal glasses and metallic films," Philosophical Transactions of the Royal Society of London, vol. 203, pp. 385-420, 1904.

[9] G. Bader, Université de Moncton, private communication. 

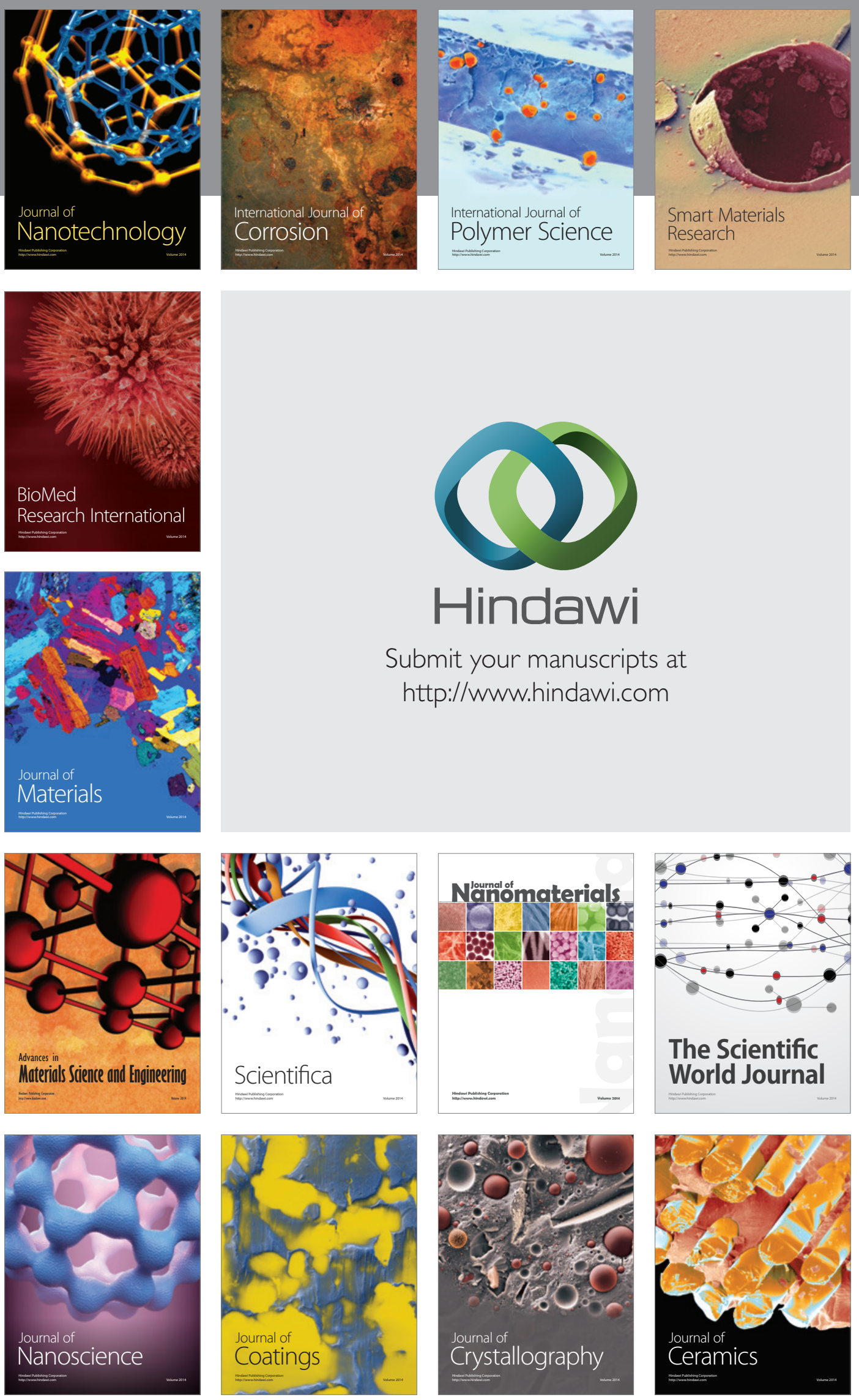

The Scientific World Journal

Submit your manuscripts at

http://www.hindawi.com

\section{World Journal}

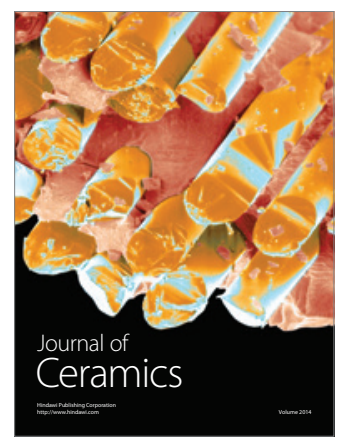

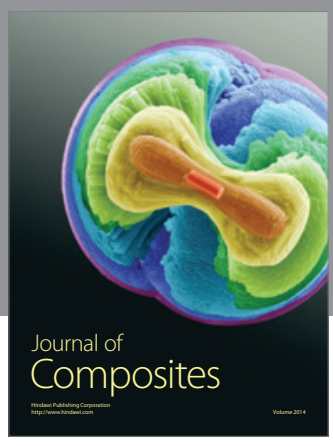
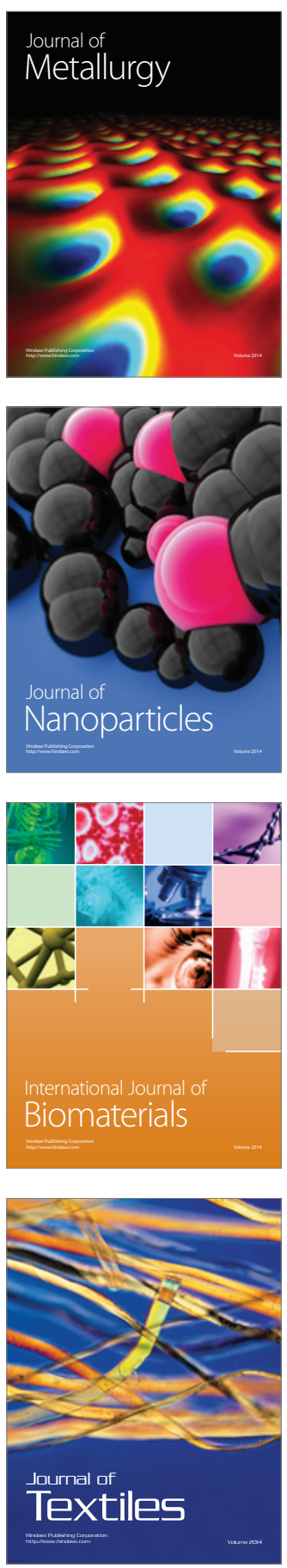